\title{
Large-scale Topographic Web Maps Using Scalable Vector Graphics
}

\author{
Peter Pavlicko \\ Dept. of Cartography, GIS \\ and Remote Sensing \\ Comenius University in \\ Bratislava, Slovak Republic \\ pavlicko@fns.uniba.sk \\ Michael P. Peterson \\ Dept. of Geography/ \\ Geology \\ University of Nebraska \\ at Omaha \\ mpeterson@mail.unomaha. \\ $e d u$
}

"For cartography, the Internet not only offers new tools for the distribution of spatial data but also new ways to present spatial data."
With the advent of the Internet, particularly the World Wide Web, the use and demand for online maps has grown very rapidly. Large scale topographic maps from the United States Geological Survey (USGS) at a scale of 1:24,000 have been traditionally distributed in paper form. To make these maps available to a larger number of people, rasterized versions are now available from various sources. Instead of the common raster format presentation, the solution presented here is based on a vector approach using Scalable Vector Graphics (SVG), a relatively new vector format describing vector graphics for the Web. SVG provides many advantages compared to the use of a raster-based presentation, such as the quality of the graphical representation, maintenance, actualization, interactivity, and extensibility through other Web programming languages. The purpose of this research is to propose an optimal and logical structure for a SVG document with a minimal file size that would be universally applicable to all USGS large scale topographic maps while maintaining the graphic quality at a comparable level with maps presented on paper. The study shows that SVG is a promising technology for delivering high quality, fully-vector topographic maps via the Internet, both in terms of graphic quality and interactivity.

Keywords: SVG, Vector Graphics, Topographic Maps

\section{Introduction}

$\mathrm{T}^{\mathrm{l}}$ he Internet has proven to be a phenomenal communication technology, bringing about new possibilities to distribute, share, and access information. For cartography, the Internet not only offers new tools for the distribution of spatial data but also new ways to present spatial data. Many authors see the Internet as the revolution in the area of cartography and GIS and point out the "irreversible" trend of this new medium (Peterson, 1999). It is estimated that more maps are distributed through the Internet than in paper form (Peterson, 2003).

The problem of disseminating spatial information through the Web is that it is a complex process involving technological, cartographic, and programming issues. Internet GIS solutions commonly make use of an underlying database to support the presentation of user-defined maps. At present, there are many Internet GIS variations that are offered by vendors. These usually work on the same general schema: database server, map server, web server and client (Peng and Tsou, 2003). However complex the underlying operation, it is the user, at his/her web browser, that is always the final consumer of the spatial information presented in map

Initial submission, June 2004; revised submission, October 2004; final acceptance, October 2004 
form. Therefore, the issue of cartographic quality plays a very important role in the process of map distribution via the Web.

Besides the inclusion of basic map features, cartographic quality of web delivered maps can be understood as a complex set of operations consisting of graphic quality, interactivity, legibility, easy maintenance and manipulation with map objects. Neumann and Isakowski (2002) point out that cartographic quality is often determined by the format in which maps are distributed. Raster formats, such as JPEG and GIF, have been widely criticized for use in Internet cartography (Neumann and Winter, 2003; Held, Ullrich, Neumann, and Winter, 2004). In general, vector formats have many advantages for web mapping applications compared to raster counterparts (Kraak and Brown, 2001). Neumann (2003) proposes that the web-client data format should (1) fulfill several requirements such as superior graphical quality and interactivity; (2) be based on standards and XML; (3) not be a proprietary format; (4) allow scripting, extensibility and animation; and (5) be available for all major operating systems and browsers. A relatively new vector standard for Web graphics called SVG (Scalable Vector Graphics) can fulfill such requirements.

Most of the maps available through the Web are thematic maps, or small general reference maps. Although there are some implementations for the large scale topographic maps in raster format on the Internet, such as from http:/ / www.Topozone.com, the cartographic quality and interactivity leaves much to be desired. Therefore, we examined the possibility of creating a vector, large-scale topographic map in SVG, and of exploring the advantages and the limitations of this technology.

This research proposes a method for creating USGS primary series topographic maps at a scale of 1:24,000 using the SVG format. The goal is to create an innovative way for delivering high-quality, interactive vector topographic maps via the Internet. It will be demonstrated that the Internet can be used to distribute maps of graphic quality comparable to paper maps. The approach described here can be applied not only to USGS topographic maps, but also to essentially any similar types of maps. It can also serve as a basis for the design of a database that would facilitate the distribution of a user-defined subset of a large-scale topographic map in SVG through the Internet.

\section{Theoretical and Methodological Aspects}

\section{USGS Large Scale Topographic Maps}

Topographic maps portray detailed information about the landscape of both natural and man-made features. They are an indispensable tool for government, science, industry, and leisure for a wide variety of purposes. The USGS (U.S. Geological Survey) provides topographic maps and updates the standard series maps in different scales for the entire United States. The map coverage has been completed for large scale maps $(1: 24,000)$, intermediate scale maps $(1: 50,000$ and 1:100,000) as well as small scale maps $(1: 250,000,1: 500,000$, and 1:1,000,000). Large scale topographic maps at a scale of 1:24,000, also known as 7.5-minute quadrangle maps, are the most detailed and the best known USGS maps (USGS, 2003a).

Although large-scale topographic maps have been recently replaced by The National Map (a new model for geographic base information), they remain a valuable source of information, and serve as the basis for other mapping applications (including The National Map). Like other topographic maps, large-scale topographic maps at a scale of 1:24,000 have
"Raster formats, such as JPEG and GIF, have been widely criticized for use in Internet cartography."

\author{
"This research proposes a \\ method for creating USGS \\ primary series topographic \\ maps at a scale of 1:24,000 \\ using the SVG format."
}


"Simply stated, XML is regarded as the technology on which the future Web will be based."

"It is clear that SVG is becoming a very powerful tool for presenting spatial information on the Web." been traditionally distributed in paper form. A need to distribute these maps electronically came with the advent of computers and the Internet. The topographic maps were digitized and stored in The National Digital Cartographic Data Base (NDCDB).

These maps that are the elementary part of The National Mapping Program (NMP) provided by the USGS, have been created and distributed based on standards (USGS, 2003b). Digitally, they are distributed in the SDTS standard (Spatial Data Transfer Standard). There are two USGS cartographic products in the SDTS standard; vector Digital Line Graph (DLG) and raster Digital Elevation Model (DEM) data.

There are several methods for obtaining DLG data for 1:24,000-scale topographic maps, either by downloading from the Internet, or ordering from USGS. A geodata searching tool EarthExplorer (http:/ / earthexplorer. usgs.gov) or USGS FTP server (ftp://edcftp.cr.usgs.gov/pub/data/DLG/ $L A R G E \_S C A L E /$ ) provided by USGS represents several Internet access possibilities. A series of government publications (http://topomaps.usgs. gov) provides all relevant information about topographic maps-for example, where they can be found, specific standards, data sources, and links.

\section{SVG - A Web Standard for Vector Graphics}

With the tremendous growth in the use of personal computers and the Internet in the last decade, there is a growing demand for a cross-platform, independent and standardized technology for presenting data, and for providing interoperability, evolution, and decentralization. XML (eXtensible Markup Language), originally designed and promoted by W3C, represents the answer to such demands. It is the universal standard for structured web documents, providing maximal independence of networks and platforms. It is also a key technology in the development of Web services. Simply stated, XML is regarded as the technology on which the future Web will be based.

XML legitimately found a positive response in the field of cartography and GIS in web mapping applications. For example, it was used to define a new standard that would provide spatial data encoding and interoperability called GML (Geography Markup Language). Graphic representation played an important role in this new framework. SVG, based on XML, defines a new grammar for defining $2 \mathrm{D}$ vector-based graphics for the Web and other applications.

SVG, a non-proprietary and a fully open standard that is based on $\mathrm{XML}$, incorporating the advantages of XML technology, has brought a new light to the graphic representation in terms of graphic quality, interactivity and extensibility through other programming languages. The features and syntax for SVG is defined by W3C in the Scalable Vector Graphics (SVG) 1.1 Specification (SVG, 2003). Currently, there are many practical web mapping applications using SVG available online. They differ in technological implementation and the intended platform. It is clear that SVG is becoming a very powerful tool for presenting spatial information on the Web.

The advantages of SVG for web cartographic purposes include:

- It defines vector graphics

- It is not proprietary, but rather an open standard defined by W3C

- An XML-based technology compatible with other XML standards 
- Text-based format, easily readable and editable by simple text editors

- Compatible with CSS, allowing for the definition of presentation attributes

- DOM access, allowing customization with JavaScript

- Extensibility with other server technologies like Java, ASP, PHP, PERL etc.

- Flexible implementation on different platforms and operating systems

\section{Bringing Large Scale Topographic Maps to the Web}

Online, large scale topographic maps provide many advantages for users. For example, because the information is provided through a server, a user would not have to rely on his/her software to process and display such information. A user may select a particular area of interest, and such information is available anywhere and anytime if the Internet connection is provided. Combined with interactive features, one could even analyze online topographic maps leading to improved decision-making. Maps provided in such a way could be used for any application that requires fast access and very detailed spatial information.

There are a number of alternatives for delivering large scale topographic maps through the Web, ranging from the simple to the very sophisticated. Static map images in raster format, even if they are scanned depictions of paper topographic maps or exported from a program in a raster format, represent the simplest form of maps for the Web. Different raster formats are used for these types of images such as JPEG, GIF, and PNG. These files can be embedded either inside an HTML page, or disseminated separately as raster images since a web browser is able to display them. This solution does not offer many possibilities for interactivity, and for the incorporation of dynamic features.

An Internet-enabled GIS represents another way to distribute maps via the Internet. While such a system requires a large server and sophisticated software, it can offer an intelligent solution for dissemination of highly interactive and dynamic maps. A database, where geospatial data is stored, plays a central role in such an implementation. However, these solutions do not always represent the best possible approach for map dissemination in terms of graphic quality, interactivity, and flexibility. At the present time, most commercial GIS systems are mainly server-based and use expensive server/Java-applet/plug-in combinations (Neumann, 2003). Each interaction with a map requires contacting a database server. This process is quite time-consuming and results in undesirable delays when querying or examining objects. Furthermore, the large-amount of client-server traffic burdens map-servers and bandwidth. Solutions based on client-side architecture using open web standards technology like SVG can bring these types of maps to the Web in an effective, inexpensive way with reliable quality. Neumann (2003) describes a procedure in which large-scale topographic maps can be delivered using SVG and open-source database technology.

There are several sites that distribute USGS large-scale topographic maps via the Internet-at present; http:/ / www.Topozone.com is perhaps the best known. Topozone's architecture is server-side, using the open source MapServer program, and the resultant maps are in raster format.

\section{A Case Study - Online Large Scale Topographic Maps using SVG}

This study focuses on the final representation of large-scale topographic maps in SVG, rather than on proposing a complex architecture for an

\section{"Online, large scale \\ topographic maps provide \\ many advantages for users."}

"Solutions based on client-side architecture using open web standards technology like SVG can bring these types of maps to the Web in an effective, inexpensive way with reliable quality." 
"The findings would be very useful for a database application that would deliver high-quality topographic maps on demand."

\footnotetext{
"The Digital Line Graph (DLG) represents the initial source of digital data for USGS large scale topographic maps."
}

Internet GIS solution to deliver topographic maps. The aim is to create an online topographic map that is visually similar or even identical with the paper version, and to propose a logical document structure that is designed for further application, such as the inclusion of interactive features. The findings would be very useful for a database application that would deliver high-quality topographic maps on demand. Several steps are involved in converting existing digital topographic maps to the SVG format. These steps include (1) the processing and conversion of source data to SVG; (2) the optimization of the SVG document structure; (3) the creation of map symbology according to accepted standards; and (4) the addition of interactive features.

\section{Processing and Conversion of Source Data to SVG}

The Digital Line Graph (DLG) represents the initial source of digital data for USGS large scale topographic maps. SVG is a relatively new format, so such conversion methods have not yet been developed. Because there are no tools available for direct conversion of this file format to SVG, other methods for converting DLG to SVG have to be used.

Most GIS systems import DLG files, including both graphic and attribute data. Once imported, data can be processed, analyzed, and converted to various vector formats. Most of current desktop GIS programs also offer export possibilities to SVG. This means that DLG can be easily imported, processed, and exported to SVG using a GIS program. However, there are still several shortcomings in this process. Geometric (topological) properties of the DLG files have inappropriate impact on the resultant SVG graphics. In addition, the SVG code produced by GIS programs usually requires modifications and optimizations to the most appropriate document structure. Symbolization of map features, especially for more complex symbols, is also not very well supported. The reasons for using a GIS program for processing source DLG data can be summarized in three points:

- There are no direct import/export tools from DLG to SVG (using another program is necessary).

- Processing the source data is necessary in order to separate map objects and eliminate inappropriate topological structure of map objects (unneeded number of vertices increases the file size). The illustration on left in Figure 1 displays a line from a DLG file as multiple segments. The SVG file on the right combines these segments into a single line without any breaks that interrupt graphics.

- The separation and export of individual map objects (roads, contours, rivers, etc.) is necessary in order to assign their graphic attributes (style and symbology).

The method of processing and converting source data to the SVG structure is depicted on the Figure 2. The figure demonstrates how DLG files, distributed in nine basic thematic layers, have to be imported, processed, and separately exported to the final SVG structure according to their geometric and thematic properties/affiliation. For example, spot heights (point features) and contours (line features) represent the different geometry (point/line) but the same thematic layer: hypsography. The purpose of applying this procedure is to separate individual objects in order to assign their symbology as well as prepare these individual objects for further use. This means creating the logical structure of the final SVG document. 


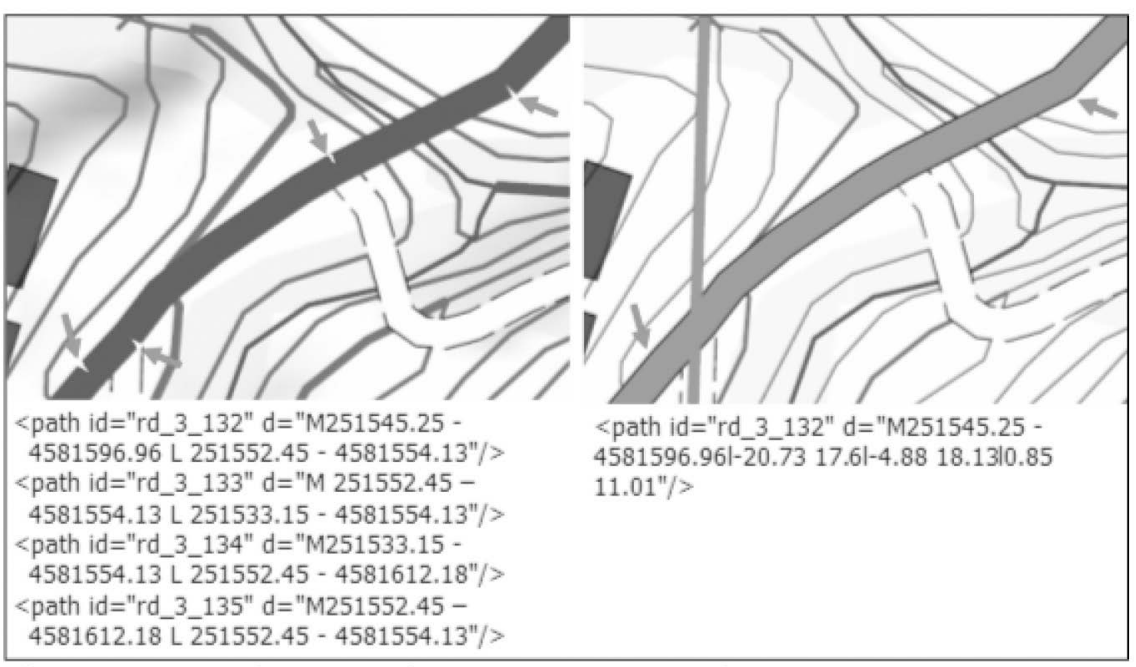

Figure 1. Inappropriate topological structure of DLG objects - unneeded number of vertices and breaks (on the left) instead of one solid line (on the right) - that have impact on the graphical representation and amount of SVG code. (see page 61 for color version)

Conversion options for exporting processed data from a GIS program to SVG differs between most commonly used GIS programs. Conversion tools offer good solutions but they still have many limitations. For example, they cannot convert more complex features such as multi-lines (a line that consists of more than one line), and symbols, that would be necessary for the creation of a large scale topographic map according to the standard symbology (USGS, 2003c).

\section{SVG Document Structure}

Once all map objects are encoded in separate SVG files, the process of compacting and creating the SVG document structure can be defined. The focus in this process is placed on two aspects, the optimal and logical file
"Conversion options for exporting processed data from a GIS program to SVG differs between most commonly used GIS programs."

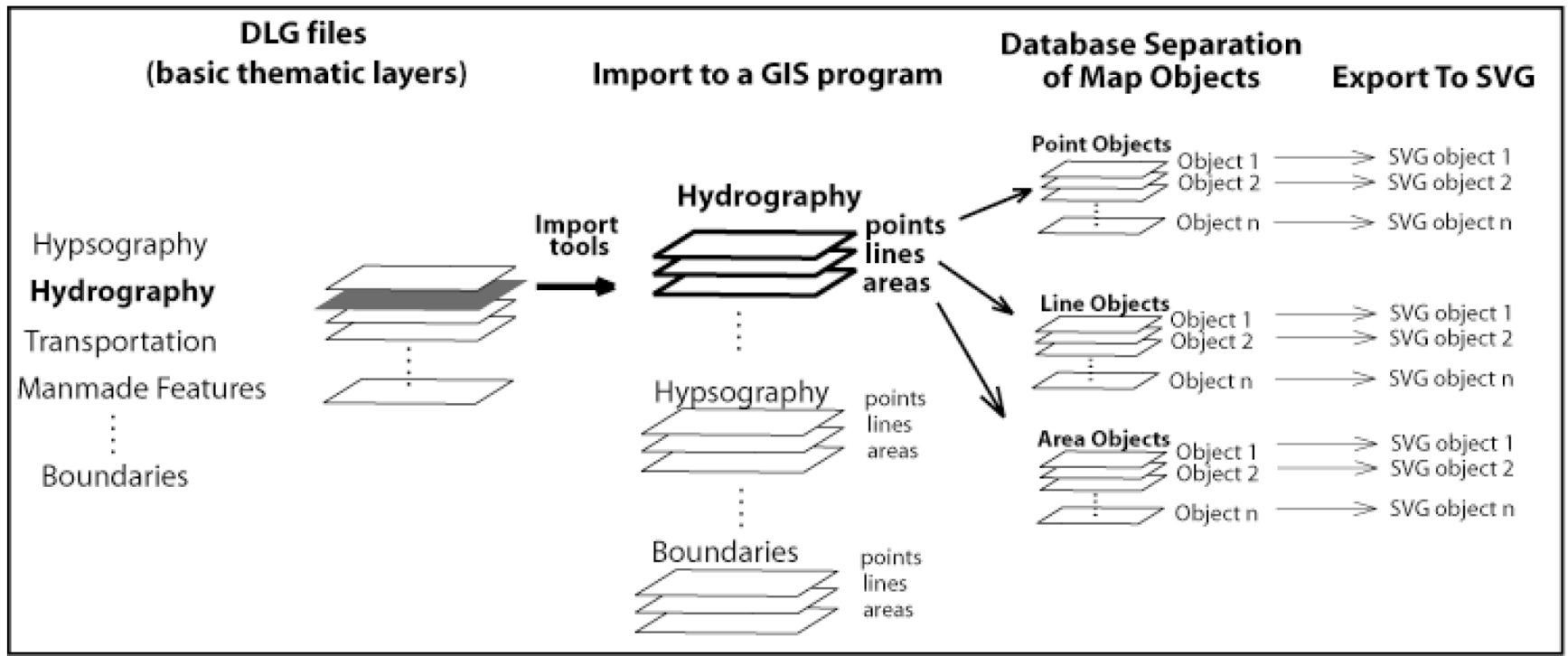

Figure 2. The process of importing, processing, and exporting source data from DLG to SVG on the example of the hydrography thematic layer. 
"Since the SVG topographic map will be distributed via the Internet, the size of the resultant file should be as small as possible."

"The structure of the SVG document has to fulfill certain hierarchical object order..." structure that reduces the file size to a minimum and also the appropriate symbology of large scale topographic maps.

Since the SVG topographic map will be distributed via the Internet, the size of the resultant file should be as small as possible. Therefore, the structure of the code is important as it directly determines the file size. Each topographic map consists of a series of map objects that are represented by symbols. These symbols need to adhere to USGS standards. The process of SVG code optimization and minimization is divided into two parts. First, the structure of the SVG document is proposed. The second part discusses the cartographic symbols that have to be created in order to create the final map with the standard symbology.

Many things need to be considered to create a completely vector topographic map in SVG. For example, how map objects will be grouped together, what is the rendering order of the SVG document and how effectively these objects are encoded in SVG need to be given careful consideration. The topographic map contains several thematic layers including area, line, point, and text features. To avoid unnecessary overlap when objects are rendered, the order of area, line, and point objects among all thematic layers has to be established. According to SVG Specification 1.1, elements in a SVG document fragment have an implicit drawing order, with the first elements in the SVG document fragment getting "painted" first. Subsequent elements are painted on top of previously painted elements. SVG grouping elements, such as the ' $\mathrm{g}$ ' tag, have the effect of producing a temporary separate canvas initialized to transparent black onto which child elements are painted (SVG, 2003).

The proposed SVG structure builds from the assumption that area features have to be rendered first, which means that they also have to be defined first in the document. Line features are then second, followed by point and text features. Moreover, the area, line, point, and text features that relate to the same thematic layer have to be organized in order to provide the proper rendering. The structure of the SVG document has to fulfill certain hierarchical object order (basic cartographic rules) and
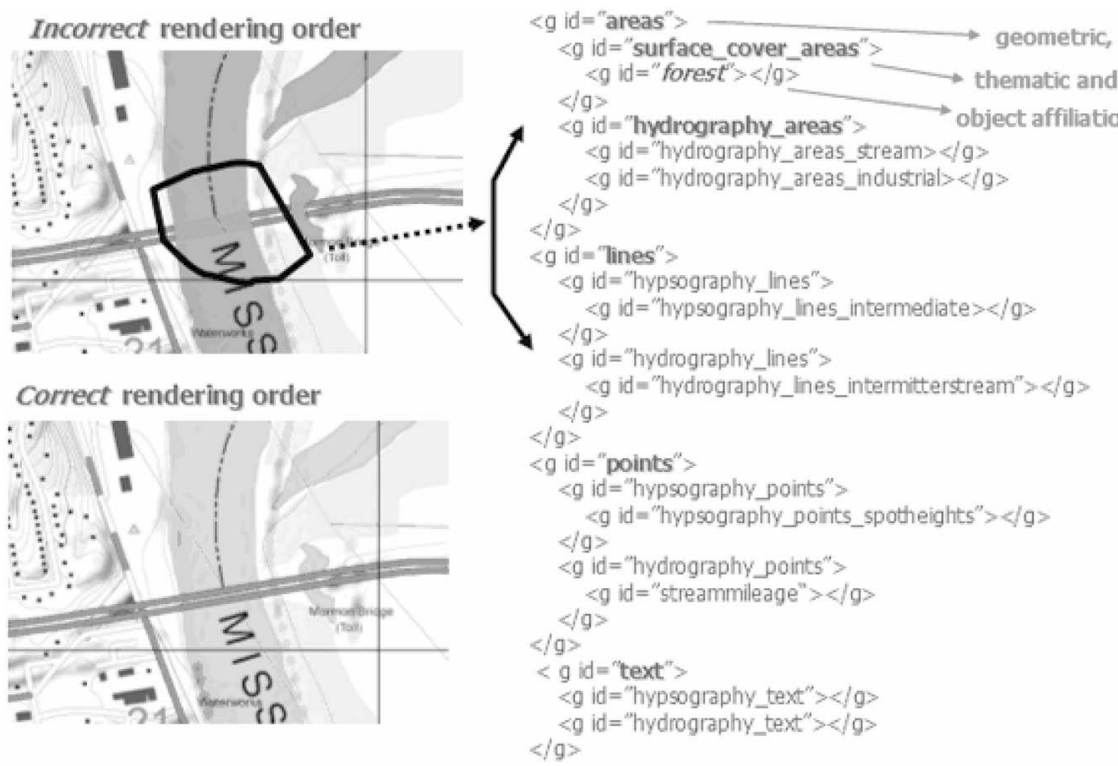

Figure 3. Example of the "overlap problem" caused by an incorrect hierarchical rendering object order with the proposed correct SVG code structure. (See page 61 for color version) 
rendering order to avoid problems with display. It has been proposed as follows:

- All objects that represent the same map object are grouped, and create the lowest hierarchical level of the SVG document (e.g., all churches as map objects are grouped to create a group "church").

- All objects that relate to the same category (thematic layer) are grouped together based upon their geometry, and create the next hierarchical level. For example, all area objects that relate to hydrography are grouped together, or all point features for manmade features are also grouped together.

- Groups (objects) that represent all categories and relate to the same geometry are grouped to the highest hierarchical level (e.g., all thematic layers with area features are grouped together).

- The rendering order for the groups with highest hierarchical order is as follows: areas are defined first, followed by lines, points and then text.

- The rendering order for the different thematic categories with the same geometry is established according to their affiliation to the real surface (e.g., contours should be rendered first in relation to roads or railroads because they represent the surface, and roads are built on surface).

Another aspect that deals with the SVG code optimization is about possibilities for its minimization. Suggestions proposed by Neumann and Isakowski (2002) lead to intensive code minimization. To minimize code, relative instead of absolute coordinates were implemented, geometry was re-used when applying two or more different styles to one object, and symbols were centrally defined with styles.

\subsection{Map Symbology}

Cartographic symbols on 7.5-minute quadrangle maps are defined by USGS Standards (USGS, 2003c). Each symbol is defined by its size, color, placement, number, and name. Based upon these standards, it is possible to create the same symbology for map objects in SVG that are found in the paper version of topographic maps.

SVG offers many advantages for the creation of symbology for 1:24,000scale topographic maps. Symbols can be applied to map objects in two different ways. According to a level of symbol complexity, symbology or style can be applied to a map object either directly by styles, or by using predefined symbols. The application of the SVG "style" to map objects as SVG elements is not a complicated process, and there are a variety of ways to handle such definitions. SVG offers four methods of applying style to elements:

- XML presentation attributes

- Inline CSS (Cascading Style Sheets) properties

- Internal CSS style sheet references

- External CSS style sheet references

Direct application of style (using the first two methods listed above) allows for the quickest method to test the results of style application, whereas style sheets (internal or external) allow for cleaner, more compact, and more organized documents. Since this effort is directed towards

\author{
"To minimize code, relative \\ instead of absolute coordinates \\ were implemented. . ."
}

"Based upon these standards, it is possible to create the same symbology for map objects in $S V G$ that are found in the paper version of topographic maps." 
"For example, a point feature, like a church or a spot height, is defined as a symbol and then it can be later referenced as many times as needed."

"Interactive features implemented in the SVG map extend the map possibilities." smaller file sizes for the SVG document, CSS style sheets provide a better solution. They make the document clearer and reduce the amount of code.

One of the most useful features in SVG is the ability to establish an object (or group of objects) as a symbolic group ('symbol' tag). By doing so, this "symbol" can be referenced multiple times throughout the document by a 'use' tag. This is useful especially for point features. For example, a point feature, like a church or a spot height, is defined as a symbol and then it can be later referenced as many times as needed. For complex map features, such as multi-lines, which consist of several different lines with different styles, a method suggested by Neumann and Isakowski (2002) leads to a very effective solution. To create a multi-line, they propose to re-use the geometry of a single line. This is useful for instance in case of roads (class1 and class2) where lines consist of two or three styles. Figure 4 displays the code and corresponding graphic example:

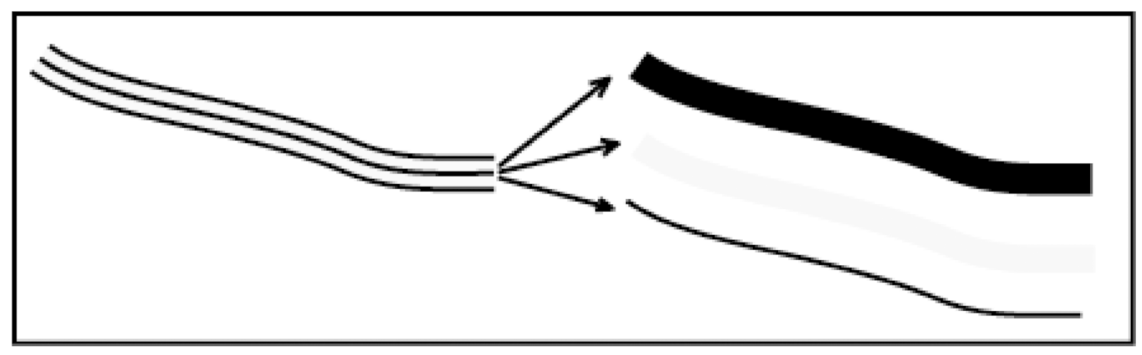

Figure 4. Multi-line composed of three styles as proposed by Neumann and Isakowski (2002). (See page 62 for color version)

Symbology for USGS large-scale topographic maps can be defined universally as a symbol library and used for any map of this type. This can be done by an external CSS file. Names of symbol classes defined in CSS should follow the "National Mapping Program Technical Instructions - Part 6, Publication Symbols (USGS 2003c)" and the particular number under which specific symbol can be found. Names should also include their geometric (area, line, point or text), and thematic (hypsography, hydrography, and so on) affiliation, based upon the rendering order in the SVG document. This could be an example of a symbol style:

hp300line - where hp refers to the thematic affiliation (Hypsography), 300 refers to its number in the "National Mapping Program Technical Instructions - Part 6, Publication Symbols," and line refers to its geometric property.

\section{Interactivity}

Interactivity represents an important method to allow users to better extract topographic information from a web map. The number of such interactive features that can be implemented is basically unlimited and can be developed to serve specific applications. Zooming, turning layers on and off, providing coordinate readouts, and displaying attributes through mouseover functions represent basic functions that would allow interactive manipulation with a topographic map.

Interactive features implemented in the SVG map extend the map possibilities. With client-side scripting languages like ECMAScript (JavaScript), one is able to access the features SVG and every browser feature's 


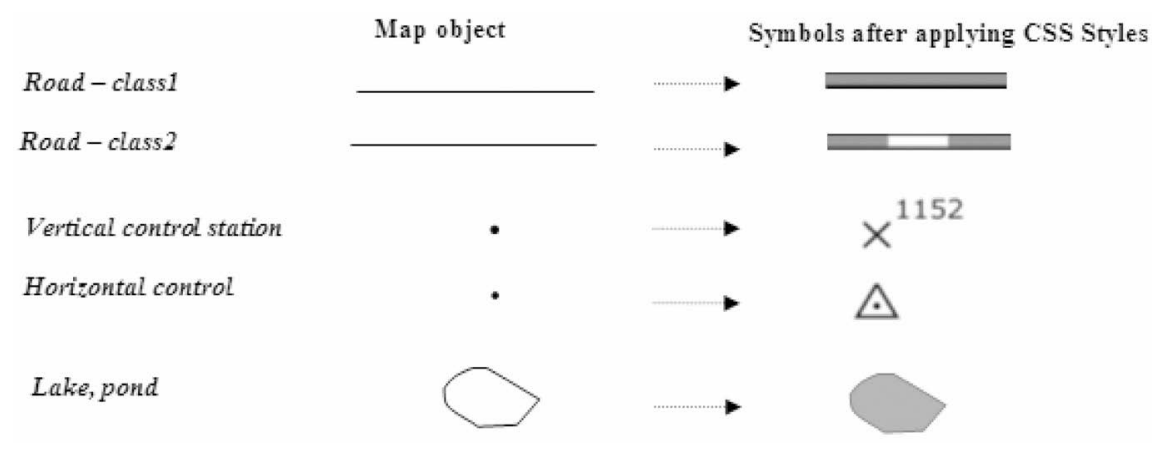

Figure 5. Applying styles using CSS to create map symbology. (See page 62 for color version)

DOM (Document Object Model). Basic interactivity with the SVG map, such as zooming, panning, and resetting (returning to the original view) can be provided through the SVG Viewer, as it is currently done with Adobe's SVG Viewer (the most popular SVG viewer). However, these functions could be implemented separately from the viewer in a more sophisticated form. Using client-side scripting languages like ECMAScript, one can develop many useful interactive functions. For example, Neumann and Isakowski (2002) propose a way to implement interactive features with a SVG topographic map. They also provide a list of features that can be useful for interactive topographic web maps. Besides basic functions that serve navigational purposes (e.g., coordinates, zooming, layers on/off), they propose other functions that would allow analyzing a topographic map through the use of ECMAScript (JavaScript), such as linked elevation profile, interactive hill shading, displaying attribute data, etc.

\section{Results and Discussion}

An online example of a SVG 1:24,000 topographic map with several interactive features is available at http://ptolemy.unomaha.edu/ pavlicko/. This project shows the possibilities of SVG for bringing this type of map to the Web (Figure 6). This SVG implementation was created using openstandard and open-source tools including GRASS GIS, SVG, XHTML and JavaScript (ECMAScript). SDTS data (DLG and DEM) provided by USGS served as the initial source of data for this project. Data, as it was presented in previously, was processed and exported to SVG and manually edited to the final file structure. Besides the static graphical map in a vector form, several interactive features such as toggling thematic layers "on" and "off," and UTM coordinate readout for X, Y and Z (elevation) were developed using ECMAScript.

Only a portion of a single 1:24,000 topographic map was coded in SVG. Creating an optimal file size for the Web is a very limiting factor. The information content that such maps depict, including all thematic layers, is rich, and this results in large files. The portion of the whole 7.5-minute quadrangle represented in SVG is shown in Figure 7. The size of that SVG file, not including ECMAScript coding, is $940 \mathrm{~KB}$ in uncompressed form or approximately $300 \mathrm{~KB}$ in compressed form (gzip). Depending on the connection speed, such a file in compressed form would be downloaded in approximately 10 seconds with the slowest modem connection $(28.8 \mathrm{kB} / \mathrm{s})$.

File sizes for whole topo-sheets in SVG (all thematic layers) would vary, depending on the information content of the map (e.g., number of

\author{
"Basic interactivity with the \\ SVG map, such as zooming, \\ panning, and resetting \\ (returning to the original view) \\ can be provided through the \\ SVG Viewer ..."
}

"This SVG implementation was
created using open-standard
and open-source tools including
GRASS GIS, SVG, XHTML
and JavaScript (ECMAScript)." 
"We propose that, at a maximum, approximately only one quarter of a topo-sheet is appropriate for presentation through the Web."

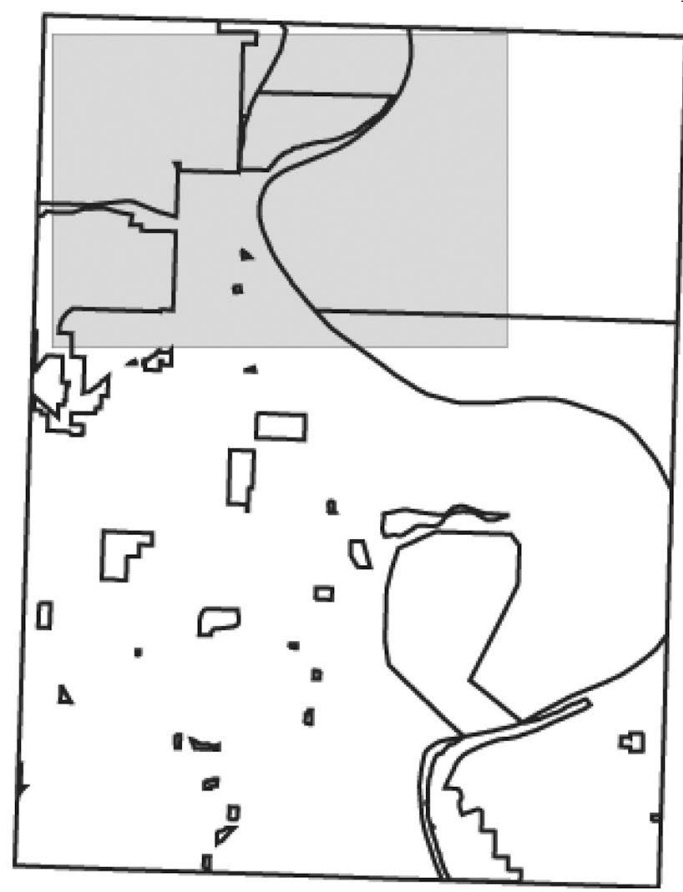

Figure 7. The area of the SVG map project (grey color) on the 7.5-minute quadrangle map (North Omaha, NE). The area represents about $1 / 3$ of the map.

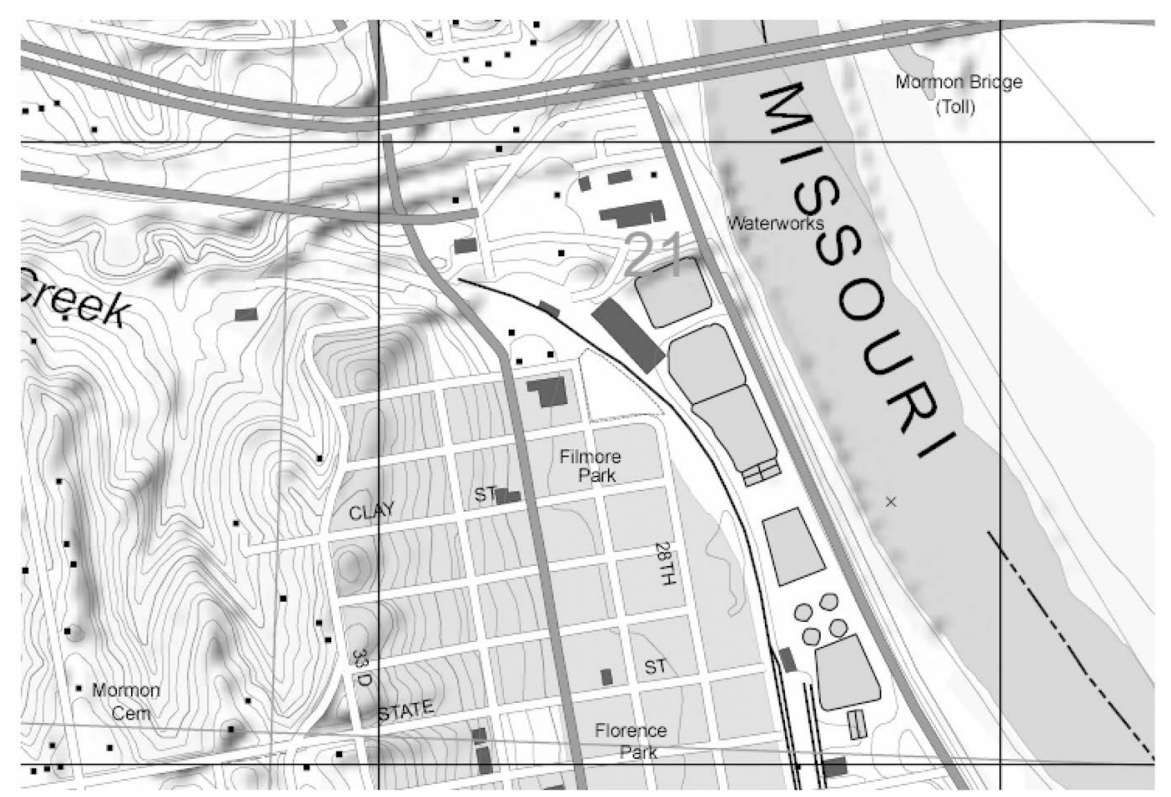

Figure 6. SVG provides considerable quality of graphics (detail associated with SVG). (See page 63 for color version)

contours). In order to investigate SVG file sizes, research was conducted on several 1:24,000-scale topographic maps from Wyoming, Montana, and Nebraska. These SVG files were all between 3 to 4 megabytes in size. Such large files are not appropriate for the Web at this time, but would be feasible through Point-to-Point (P2P) protocols currently implemented with music files. We propose that, at a maximum, approximately only one quarter of a topo-sheet is appropriate for presentation through the Web.

To distribute topographic maps in SVG for a larger area that may encompass many map sheets, it would be necessary to create a database that could distribute small parts of an individual topographic map on demand. Methods for delivering larger geographic datasets for large scale topographic maps are proposed by Neumann (2003).

\section{Conclusion}

This study has demonstrated that Scalable Vector Graphics (SVG) is a promising vector technology for delivering high quality interactive topographic maps via the Internet. Vector formats, in comparison to their raster counterparts, provide many advantages such as the quality of the graphical representation, maintenance and actualization, interactivity, and extensibility through other web programming languages. The corresponding implementations for the distribution of such maps in raster form leave much to be desired in graphic quality as well as interactivity.

This work has shown that it is possible to present portions of a USGS 7.5-minute quadrangle map through the Internet in SVG. This new graphic format shows great promise for the distribution of this type of map. The graphic quality and manipulation possibilities with such SVG topographic maps are far superior to corresponding raster implementations. 
However, there are still some limitations to SVG that need to be taken into consideration. The sizes of the resultant SVG files are large. According to the results, only a portion of 1:24,000-scale topographic map sheet can be distributed, with some interactive elements. To distribute topographic maps for a larger area or encompassing many map sheets using SVG, it would be necessary to create a database that could distribute small parts of an individual topographic map on demand.

The incorporation of interactive features using client-side scripting languages like JavaScript can promote more analytical uses of these maps. The number of such features that could be implemented is basically unlimited and can be developed to serve specific applications. This represents the future challenge and an interesting subject for future research.

Held, G., Ullrich, T., Neumann, A., Winter, A.M., 2004. Comparing .SWF (ShockWave Flash) and .SVG (Scalable Vector Graphics) file format specifications. Available [Online]: http://www.carto.net/papers/svg/ comparison_flash_svg/

Kraak, M.J. and Brown, A., 2001. Web Cartography: Developments and Prospects. London: Taylor and Francis.

Neumann, A. and Isakowski, I., 2002. Interactive Topographic Web Maps Using SVG. Proceedings from SVG Open Conference, Zurich 2002. Available [Online]:

http://www.svgopen.org/2002/papers/isakowski_neumann_svg_for_ interactive_topographic_maps /

Neumann, A., 2003. Delivering Interactive Topographic Web-maps using Open-Source Database Technology. Proceedings from SVG Open Conference, Vancouver 2003. Available [Online]: http:/ / www.svgopen.org/2003/papers/abstract_neumann_topographic_maps_served_by_spatial_databases/index.html

Neumann, A. and Winter, A., 2003. Vector-based Web Cartography: Enabler SVG. Available [Online]: http://www.carto.net/papers/svg/index_ e.sthml

Peng, Z-R. and Tsou, M-H., 2003. Internet GIS. New York: John Wiley \& Sons.

Peterson, M., 1999. Trends in Internet Map Use: A Second Look. Proceedings of the 19th International Cartographic Conference and 11th General Assembly of ICA, Ottawa, Canada, Vol. I:571-580.

Peterson, M. P., 2003. Foundations of Research in Internet Cartography. In Maps and the Internet (ed.) Peterson, M. P., Amsterdam: Elsevier Press.

SVG, 2003. - Scalable Vector Graphics (SVG) 1.1 Specification. Available [Online]: http:/ /www.w3.org/TR/SVG11/

USGS, 2003a. Topographic Mapping. Available [Online]:

http:/ / erg.usgs.gov/isb/pubs/booklets/topo/topo.html
"To distribute topographic maps for a larger area or encompassing many map sheets using SVG, it would be necessary to create a database that could distribute small parts of an individual topographic map on demand."

\section{REFERENCES}


USGS, 2003b. National Mapping Program Standards. Available [Online]: http://geography.usgs.gov/standards/index.html

USGS, 2003c. National Mapping Program Standards, Primary Series Quadrangle Map Standards. Available [Online]: http:/ / rockyweb.cr.usgs.gov/nmpstds/qmapstds.html 